\title{
A New Approach 160-bit Message Digest Algorithm
}

\author{
Priteshwar Nath Sallam \\ School of IT, Rajiv Gandhi \\ Technical University Bhopal, \\ (M.P) India
}

\author{
Jitendra Agrawal \\ School of IT, Rajiv Gandhi \\ Technical University Bhopal, \\ (M.P) India
}

\author{
Santosh Sahu \\ School of IT, Rajiv Gandhi \\ Technical University Bhopal, \\ (M.P) India
}

\begin{abstract}
This paper introduces a new approach of MD Algorithm for security design. This approach comprises of the 160-bit hash algorithm for secure message digest. The results show that the 160-bit Message Digest Algorithm code is more secured than 128-bit Message Digest Algorithm code. This effort can alternate efficiently the accessible Message Digest Algorithm and hash function implementations for security design, so this approach proves a high security step towards design.
\end{abstract}

\section{Keywords}

MD Algorithm; Hash Function; Data Integrity;

\section{INTRODUCTION}

The software is designed for security purpose. A hash value computes a permanent length output called the message digest from an input message of different lengths. The MD5 message digest algorithm was developed by Ron Rivest at MIT. Until the last few years when both burst force and cryptanalytic concerns have arose, MD5 was most widely used secure hash algorithm. It is a widely-used 128-bit hash function, used in various applications Including SSL/TLS, IPSec, and many other cryptographic protocols. The MD5 algorithm breaks a sleeve into 512 bit input blocks. Every block is run from side to side a series of functions to produce a exceptional bit hash value for the sleeve[1].This paper explain how to design MD 160 algorithm and how MD 160 is more secure than past 128 bit hash algorithms.

\section{HASH FUNCTION}

A hash function $\mathrm{H}$ is a transformation that takes a variablesize input $\mathrm{m}$ and proceeds a fixed-size string, which is called the hash value $h$.Hash functions with just this property have a variety of general computational uses, but when working in cryptography the hash functions are regular chosen to have some supplementary properties. This is a contract in lots of programming languages that allocate the user to dominate equality and hash functions for an object, that if two objects are the same their hash codes must be the same. Hash functions compress a $\mathrm{n}$ (arbitrarily) large number of bits into a small number of bits.

The hash function properties are:-

- Output does not reveal information on input.

- Hard to find collisions (different messages with same hash).

- One way cannot be reversed.

\section{STRENGTH OF MD 160}

Every hash function with more inputs than outputs will essentially have collisions. This hash function MD-160 that generate 160 bits of output from an randomly large input. Since it must generate one of $2^{160}$ outputs for each member of a much larger set of inputs, the pigeon hole opinion guarantees that some inputs will hash to the same output. Collision conflict doesn't mean that no collisions exist; simply that they are hard to find. The birthday "paradox" spaces an upper bound on collision conflict: if a hash function generate $\mathrm{N}$ bits of output, an attacker who computes "only" $2 \mathrm{~N} / 2$ hash operations on arbitrary input is likely to find two matching outputs. If there is an easier method than this brute force attack, it is typically measured a flaw in the hash function. Cryptographic hash functions are usually considered to be collision resistant. But many hash functions that were once thought to be collision resistant were later broken. MD5 and SHA-1 in exacting both have published approaches more efficient than brute force for pronouncement collisions. However, some compression functions have a proof that pronouncement collisions are at least as difficult as some hard mathematical problem (such as integer factorization or discrete logarithm). Those functions are called provably secure.

\section{DATA INTEGRITY}

Data integrity assertion and data basis authentication are important security services in an economic statement, electronic business, electronic mail, software distribution, data storeroom and so on[3]. The messages are broadest of verification within computing systems Encompasses uniqueness authentication, meaning source Authentication and message contented authentication. One that deals with individual message only without regard to large context generally provides protection against message modification only. Data integrity is compulsory within a record at its design steps. from beginning to the end use of standard rules and procedures, and is maintained through the use of error inspection and validation routines[3].

Data integrity divided are four categories:-

- Entity integrity

- Domain integrity

- Referential integrity

- User-defined integrity

\subsection{Entity integrity}

Entity Integrity ensures that there are no replica reports within the table and that the field that identifies each documents within the table is matchless and never null.

\subsection{Domain integrity}

Domain Integrity mostly used for business rules. A domain is the position of all probable values for a given element. A domain integrity check usually just called a domain constraint is a rule that defines these legal values.

\subsection{Referential integrity}

Referential integrity is a main property of data which are required for one attribute. Referential integrity is a record 
thought that ensures that associations between tables remain consistent. Referential integrity works for RDBMS.

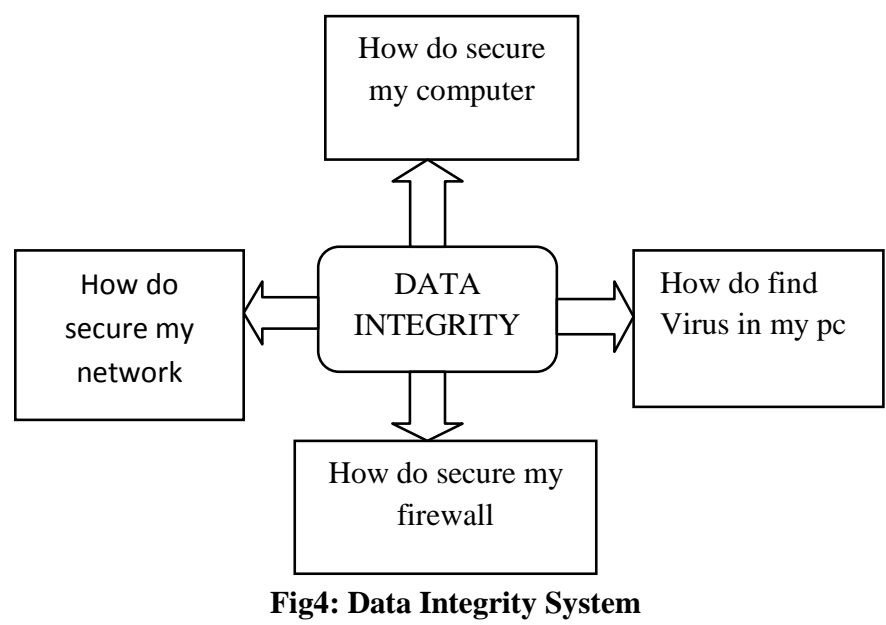

\subsection{User-defined integrity}

User Define Integrity most commonly define by user that involve in the business. User-defined integrity allows you to define precise business rules that do not descend into one of the other integrity categories.

\section{MD 160 BIT ALGORITHM}

MD 160 is an algorithm that is used to authentic data integrity from side to side the creation of a 160-bit message digest from data input that is claimed to be as exceptional to that explicit data as a fingerprint is to the explicit person. This algorithm is designed on the base of MD5 that was designed by R. Rivest in 1991. In 1993 some weaknesses in it's propose be critical exposed. In 2004 meticulous Wang et al. generated a collision for MD5, accessible at Euro Crypt 2005 in [4]. Authors in [5] and [6] then extended this collision construction method, leading to the surprising result presented in [7] at the beginning of 2009. The authors devised a convenient attack situation, where they successfully created a scoundrel digital identity certificate issued by an unconscious real-world Certification Authority (CA) and trusted by all common web browsers. The MD5 hash function was developed in 1994 developed by Professor Ronald L. Rivest of MIT, is intended for use with digital signature .Changing for one digit in any of the input blocks should have a cascading produce that finally alters the hash results[2]. MD5 algorithm is to produce a message digest of information in organize to prevent tampering. We view the entire file as a large text message, and result in a unique MD5 message digest by the irreversible string alteration method. In the future, if the stuffing of file are changed, we only recalculate MD5 message digest of this categorizer and will find the difference from the original message digest. MD5 is one of the most admired hash functions for many applications such as IPsec [9].we have enthusiastic comparison with MD5, we arbitrarily change 1 to 1024 bits in plain text in that order, and for every adjust we repeat our algorithm and MD5 each for 20 time and record the normal rate of difference in message digests. The authentication does not need to order the original data but only need to have a valuable digest to authenticate the identity of client. This reduces the prospect that original data grasped by the intruder significantly and guarantees the information security. Let's See it design from 160 bit message digest algorithm:-

\subsection{Padding Bits}

The message is "padded" (extended) so that its length (in bits) is congruent to 448 , modulo 512 . That is, the message is extended so that it is just 64 bits shy of being a multiple of 512 bits long. Padding is always performed, even if the length of the message is already congruent to 448, modulo 512.Padding is performed as follows: a single "1" bit is appended to the message, and then "0" bits are appended so that the length in bits of the padded message becomes congruent to 448 , modulo 512. In all, at least one bit and at most 512 bits are appended [11].

\subsection{Append length}

A 64-bit representation of $b$ (the length of the message before the padding bits were added) is appended to the result of the previous step. In the unlikely event that $\mathrm{b}$ is greater than $2^{\wedge} 64$, then only the low-order 64 bits of $b$ are used [11].

\subsection{Initialize MD Buffer}

A Five-word buffer (A, B, C, D, E) is used to compute the message digest. Here each of A, B, C, D, E is a 32-bit register. These registers are initialized to the following values in hexadecimal, low-order bytes first.

Word A: 01234567

Word B: $89 \mathrm{ab}$ cd ef

Word C: fe dc ba 98

Word D: 76543210

Word E: c $3 \mathrm{~d} 2 \mathrm{~d} 1 \mathrm{f} 0$

This step uses a 80 -element table $\mathrm{T}[1 \ldots 80]$ constructed from the sine function.

Do the following:

/* Process each 16-word block. */

For $\mathrm{i}=0$ to N/16-1 do

/* Copy block i into X. */

For $\mathrm{j}=0$ to $20 \mathrm{do}$

Set $\mathrm{X}[\mathrm{j}]$ to $\mathrm{M}[\mathrm{i} * 16+\mathrm{j}]$.

end $/ *$ of loop on $\mathrm{j} * /$

/* Save A as AA, B as BB, C as CC, and D as DD,E as EE. */

$\mathrm{AA}=\mathrm{A}$

$\mathrm{BB}=\mathrm{B}$

$\mathrm{CC}=\mathrm{C}$

$\mathrm{DD}=\mathrm{D}$

$\mathrm{EE}=\mathrm{E}$

/* Round 1. */

$/ *$ Let [abcd k s i] denote the operation $\mathrm{a}=\mathrm{b}+((\mathrm{a}+\mathrm{F}(\mathrm{b}, \mathrm{c}, \mathrm{d})+\mathrm{X}[\mathrm{k}]+\mathrm{T}[\mathrm{i}])<<\mathrm{s}) . * /$

/* Do the following 20 operations. *

[ABCD 07 1] [DABC 112 2] [CDAB 217 3]

[BCDA 3224$]$

[ABCD 47 5] [DABC 5 12 6] [CDAB 6 17 7] [BCDA 7 $228]$

[ABCD 87 9] [DABC 912 10] [CDAB 1017 11]

[BCDA 1122 12]

[ABCD 127 13] [DABC 1312 14] [CDAB 14 17 15]

[BCDA 1522 16]

[ABCE $\left.\begin{array}{lllll}16 & 7 & 17\end{array}\right][$ [EABC $17 \quad 12$ 18] [CEAB 18 17 19 ]

[BCEA 1922 20]

\footnotetext{
/* Round 2. */

/* Let [abcd k s i] denote the operation $\mathrm{a}=\mathrm{b}+((\mathrm{a}+\mathrm{G}(\mathrm{b}, \mathrm{c}, \mathrm{d})+\mathrm{X}[\mathrm{k}]+\mathrm{T}[\mathrm{i}])<<\mathrm{s}) . * /$

/* Do the following 20 operations. */
} 
[ABCD 15 21] [DABC 69 22] [CDAB 1114 23] [BCDA 0 $2024]$

[ABCD 55 25] [DABC 109 26] [CDAB 1514 27] [BCDA 420 28]

[ABCD 95 29] [DABC 149 30] [CDAB 3 14 31] [BCDA 820 32]

[ABCD 135 33] [DABC 29 34] [CDAB 7 14 35] [BCDA 122036 ]

[ABCE 15 37] [EABC 69 38] [CEAB 11 14 39] [BCEA 020 40]

\section{/* Round 3. */}

${ }^{*}$ Let $[\mathrm{abcd} \mathrm{k} \mathrm{s} \mathrm{t]} \mathrm{denote} \mathrm{the} \mathrm{operation}$ $\mathrm{a}=\mathrm{b}+((\mathrm{a}+\mathrm{H}(\mathrm{b}, \mathrm{c}, \mathrm{d})+\mathrm{X}[\mathrm{k}]+\mathrm{T}[\mathrm{i}])<<\mathrm{s}) . * /$

$/ *$ Do the following 20 operations. */ [ABCD 54 41] [DABC 811 42] [CDAB 11 16 43] [BCDA 1423 44]

[ABCD 14 45] [DABC 411 46] [CDAB 716 47] [BCDA 1023 48]

[ABCD 134 49] [DABC 011 50] [CDAB 316 51] [BCDA 623 52]

[ABCD 94 53] [DABC 1211 54] [CDAB 1516 55] [BCDA 223 56]

[ABCE 54 57] [EABC 811 58] [CEAB 1116 59] [BCEA 1423 60]

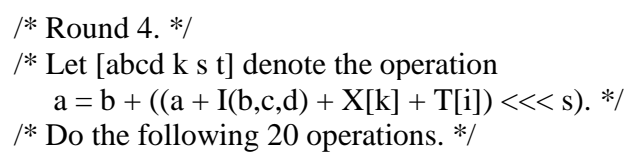

/* Round 4. */

$1 *$ Let $[$ abcd k s t] denote the operation $\mathrm{a}=\mathrm{b}+((\mathrm{a}+\mathrm{I}(\mathrm{b}, \mathrm{c}, \mathrm{d})+\mathrm{X}[\mathrm{k}]+\mathrm{T}[\mathrm{i}]) \ll<\mathrm{s}) . * /$

$/ *$ Do the following 20 operations. */

[ABCD 066 61] [DABC 710 62] [CDAB 1415 63] [BCDA 5 $2164]$

[ABCD 126 65] [DABC 310 66] [CDAB 1015 67] [BCDA 12168$]$

[ABCD 86 69] [DABC 1510 70] [CDAB 6 15 71] [BCDA 132172$]$

[ABCD 46 73] [DABC 1110 74] [CDAB 2 15 75]

[BCDA 92176$]$

[ABCE 0 6 77] [EABC 710 78] [CEAB 1415 79] [BCEA 521 80]

/* Then perform the following additions. (That is increment each of the four registers by the value it had before this block was started.) */

$$
\begin{aligned}
& \mathrm{A}=\mathrm{A}+\mathrm{AA} \\
& \mathrm{B}=\mathrm{B}+\mathrm{BB} \\
& \mathrm{C}=\mathrm{C}+\mathrm{CC} \\
& \mathrm{D}=\mathrm{D}+\mathrm{DD} \\
& \mathrm{E}=\mathrm{E}+\mathrm{EE} \\
& \text { end } / * \text { of loop on } \mathrm{i} *
\end{aligned}
$$

\subsection{Process Message in 16-Word Blocks}

This is the heart of the algorithm, which includes four "rounds" of processing. We take as input three 32-bit words and produce as output one 32-bit word [11]

$\mathrm{F}(\mathrm{X}, \mathrm{Y}, \mathrm{Z})=\mathrm{XY} \vee \operatorname{not}(\mathrm{X}) \mathrm{Z}$

$\mathrm{G}(\mathrm{X}, \mathrm{Y}, \mathrm{Z})=\mathrm{XZ} \vee \mathrm{Y} \operatorname{not}(\mathrm{Z})$

$\mathrm{H}(\mathrm{X}, \mathrm{Y}, \mathrm{Z})=\mathrm{X}$ xor $\mathrm{Y}$ xor $Z$

$\mathrm{I}(\mathrm{X}, \mathrm{Y}, \mathrm{Z})=\mathrm{Y}$ xor $(\mathrm{X} \vee \operatorname{not}(\mathrm{Z}))$

\subsection{Outputs}

The $L$ 512-bit blocks have been processed, the output from $\mathrm{L}^{\wedge}$ th age is the 160-bit message digest.

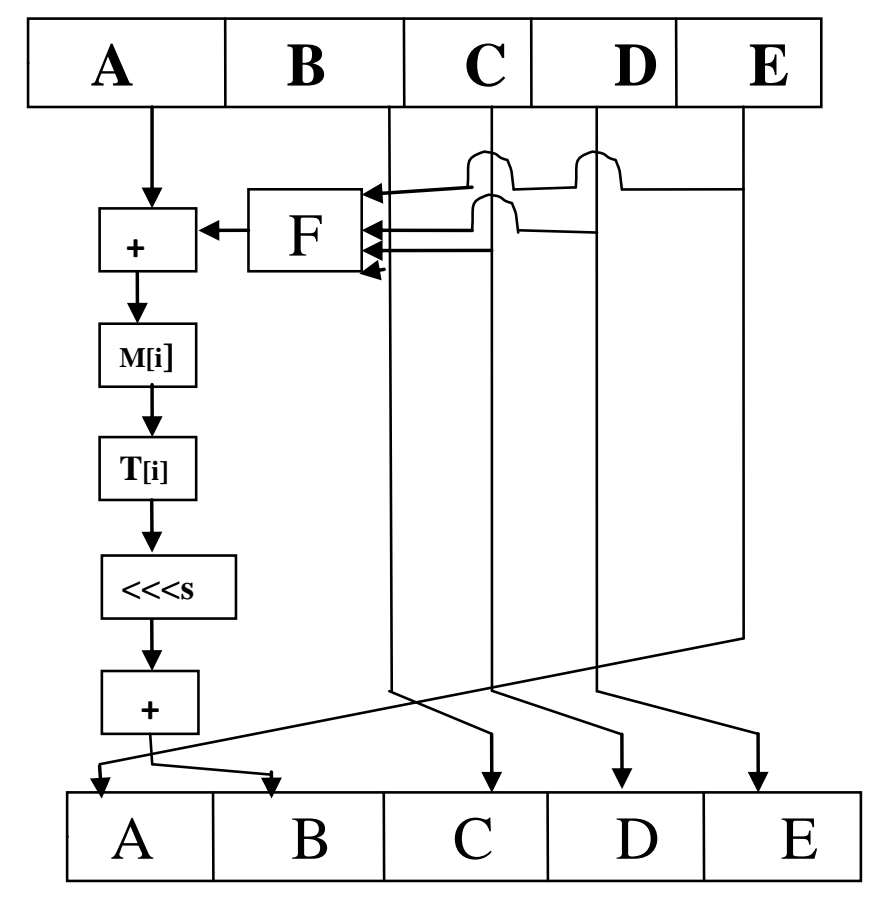

\section{RESULT}

Fig 5.5: MD 160 output

Hence when 160 bit is used in place of 128 bit Message Digest, collision is reduced and security is improved. Even this scheme is of 160 bits and need 280 bits for birthday paradox but it is strong enough for the first and second pre image attack. We can extend the length of hash to 256 or 512 bits to be more resistible against birthday attack which compared with its ancestors, is more powerful. In cryptography, a brute force attack or exhaustive key search is a strategy that can be used against in any encrypted data [15] theoretically by an attacker who is unable to take advantage of any flaws in an encryption system that would otherwise make his/her task easier.

The analysis of string by cryptool and comparison is done sequentially by the tools like entropy, floating frequency, histogram, n-gram, autocorrelation that are explained in detail in the following paragraphs.

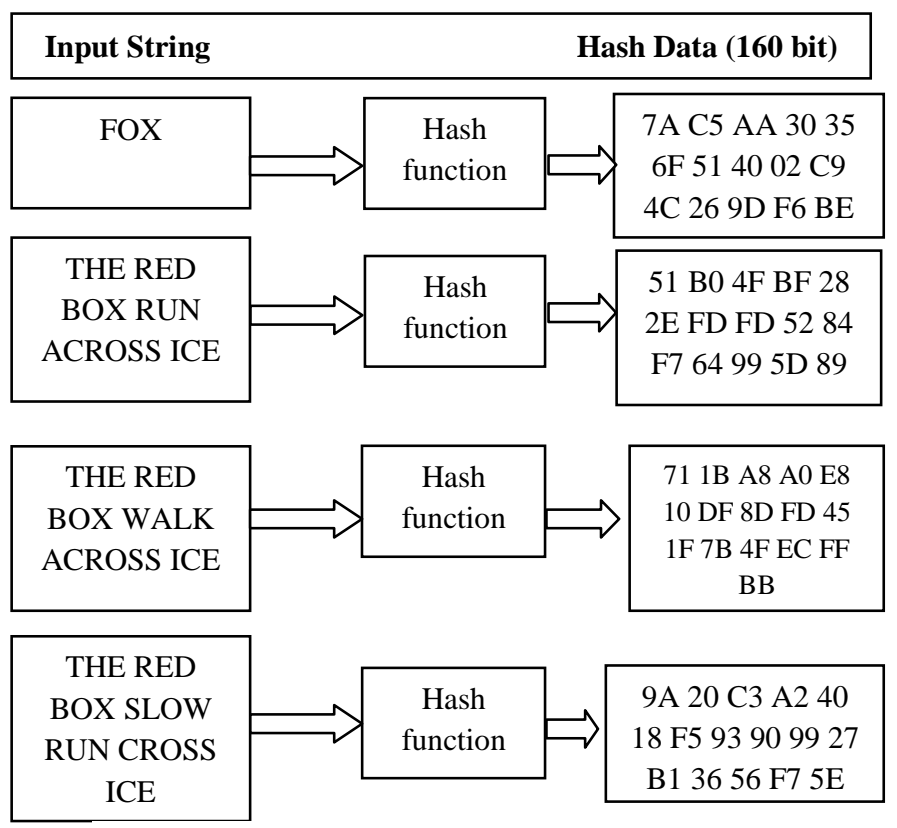

Fig 5.0: Conversion of hash value 


\subsection{Entropy}

The entropy of a source thus indicates its characteristic distribution. It measures the average amount of information which one can obtain through observation of the source or, conversely, the indeterminacy which prevails over the generated messages when one cannot observe the source.

Table 1 Calculate the entropy

\begin{tabular}{|c|c|c|}
\hline S.N & String & $\begin{array}{c}\text { Entropy (maximum } \\
\text { possible entropy 4.70) }\end{array}$ \\
\hline 1 & FOX & 1.58 \\
\hline 2 & $\begin{array}{c}\text { THE RED BOX RUN } \\
\text { ACROSS ICE }\end{array}$ & 3.65 \\
\hline 3 & $\begin{array}{c}\text { THE RED BOX WALK } \\
\text { ACROSS ICE }\end{array}$ & 3.78 \\
\hline 4 & $\begin{array}{c}\text { THE RED BOX SLOW } \\
\text { RUN ACROSS ICE }\end{array}$ & 3.80 \\
\hline
\end{tabular}

\subsection{Floating Frequency}

The floating frequency is the character which occurs most frequently. The input must contain at least 64 character(s) from the current alphabet. Calculate the all string Table 1.

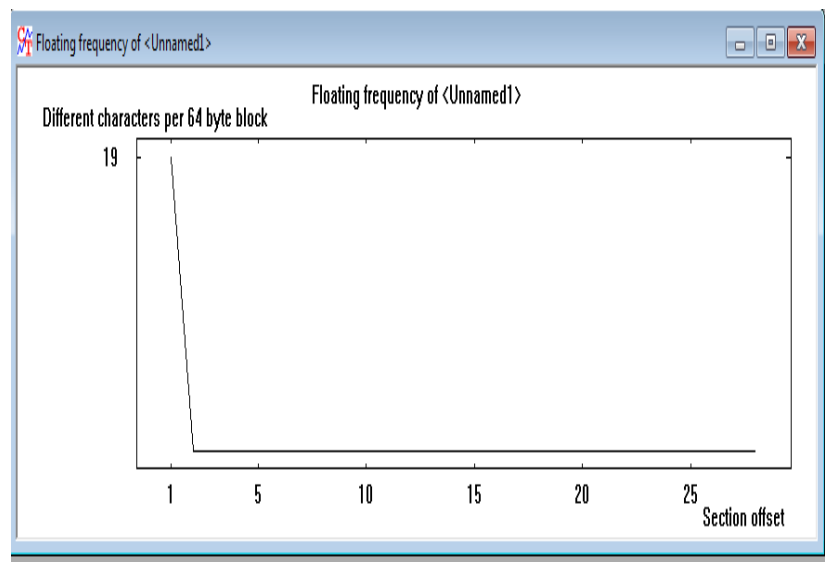

Fig 6.2: Floating frequency

\subsection{Histogram}

A histogram is one of the basic quality tools. It is used to graphically summarize and display the distribution and variation of a process data set. The main purpose of a histogram is to clarify the presentation of data.

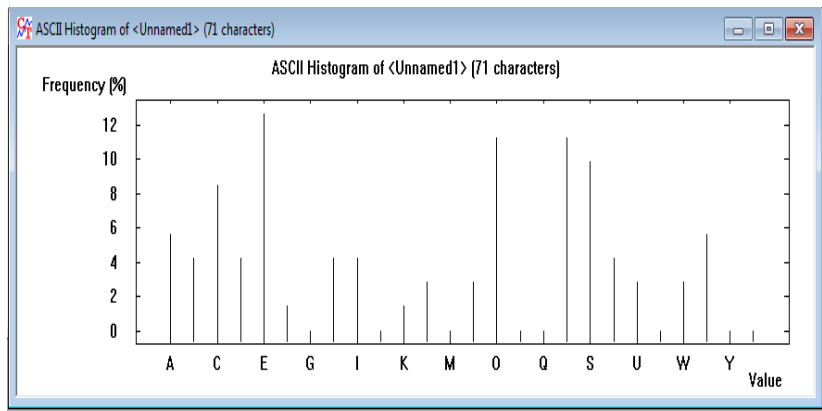

Fig 6.3: Histogram display

\subsection{N-Gram}

$\mathrm{N}$-grams are essential in any task in which we have to identify words in noisy, ambiguous input. $N$-gram is a adjacent sequence of $n$ items from a given sequence of text or speech.

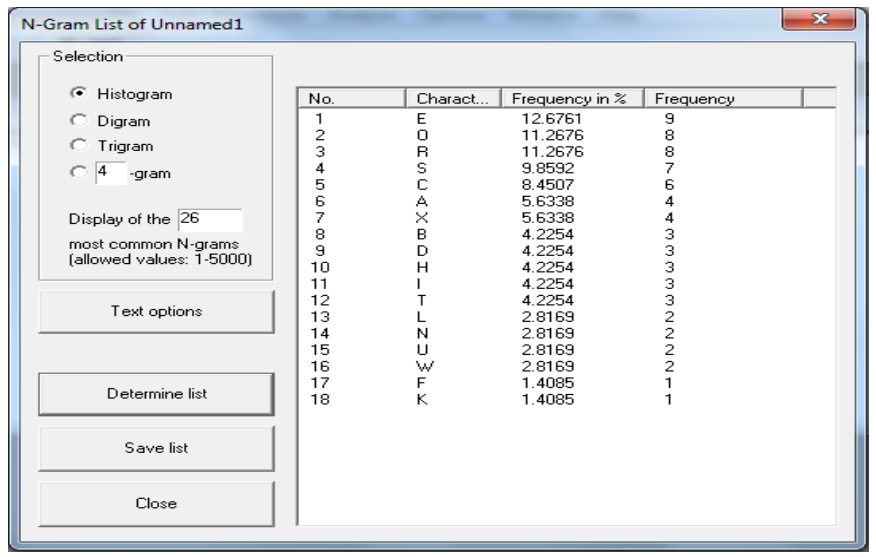

Fig 6.4: N-gram

\subsection{Auto correction}

This auto correction functionality is usually implemented by finding the string that is most similar to the given search string. The difference between two strings or minimum operation required to transform one string to another string is called edit distance between two strings. So edit distance is calculated between each string in database and given search string, the string having minimum edit distance cost is selected for the results.

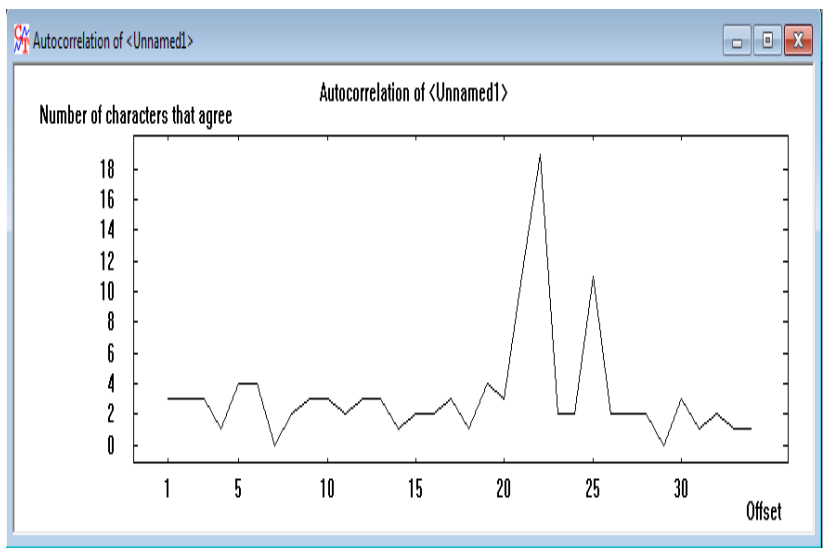

Fig 6.5 Auto correction

\section{CONCLUSION}

The simplified MD5 message-digest algorithm is simple to implement, and provides a "fingerprint" or message digest of a message of random length. It is conjectured that the complexity of two messages having the same message digest is of the order of $2^{\wedge} 64$ operations, and that the complexity of any message having a given message digest is of the order of 2^160 operations. The Message Digest 128 bit algorithm is slightly cheaper to compute, however Message Digest Algorithm 128 is currently very vulnerable to collision attacks. Message digest compresses any stream of bytes into a 160 bit value. This compression goes only in one dimension. If you give the hash of a random stream of bytes to someone, there is no theoretical way for them to go back to unique stream of bytes. 


\section{REFERENCES}

[1] R.Rivest. The MD5 Message-Digest Algorithm [rfc1321]

[2] Janaka Deepakumara, Howard M. Heys and R. Venkatesan, FPGA IMPLEMENTATION OF MD5 HASH ALGORITHM, FacuIq of Engineering and Applied Science Memorial University of Newfoundland St. John S, NF, Canada.

[3] Danyang Cao, Bingru Yang, Design and implementation for MD5-based data integrity checking system, College of Information Engineering, North China University of Technology , NCUT Beijing 100144, China

[4] X. Wang and H. Yu, "How to Break MD5 and Other Hash Functions", in: Ronald Cramer (ed.), Advances in Cryptology - EUROCRYPT 2005, vol. 3494 of Lecture Notes in Computer Science, pages 19-35, Springer,2005.

[5] M. Stevens, "On collisions for MD5", MSc Thesis,Eindhoven University of Technology, June 2007

[6] M. Stevens, A. Lenstra and B. de Weger "Chosen-prefix Collisions for MD5 and Colliding X.509 Certificates for Different Identities", in Moni Naor (eds), Science, pages 1-22, Springer Verlag, Berlin, 2007.

[7] A. Sotirov, M. Stevens, J. proceedings of Advance in Cryptology - EUROCRYPT 2007, vol. 4515 of Lecture Notes in Computer Appelbaum, A. Lenstra, D. Molnar, D.A. Osvik, B. de Weger, "MD5 considered harmful today: Creating a rogu CA certificate", 2009.
[8] Kimmo Järvinen, Matti Tommiska and Jorma Skyttä, Hardware Implementation Analysis of the MD5 Hash Algorithm, Helsinki University of Technology Signal Processing Laboratory Shenyang, China Otakaari 5 A, FIN-02150, Finland

[9] "IP Security Protocol (ipsec)," http://www.ietf.org/html.charters/ipsec-charter.html

[10] Janaka Deepakumara, Howard M. Heys and R. Venkatesan, FPGA IMPLEMENTATION OF MD5 HASH ALGORITHM, FacuIq of Engineering and Applied Science Memorial University of Newfoundland St. John S, NF, CanadaA I B 3x.

[11] MD5 Algorithm Description, http://www.cotse.com/CIE/RFC/1321/7.htm.

[12] Goldwasser, S. and Bellare, M. "Lecture Notes on Cryptography". Summer course on cryptography, MIT, 1996-2001

[13] M.M.J. Stevens (June 2007). On Collisions forMD5. http://www.win.tue.nl/hashclash/On\%20Collisions\%20fo r\%20MD5\%20-\%20M.M.J.\%20

[14] Xiaoyun Wang, Yiquin Lisa Yin, Hongobo Yu. Finding Collisions in the Full SHA-1 http://people.csail.mit.edu/yiqun/SHA1 AttackProceeding Version

[15] Christof Paar, Jan Pelzl, Bart Preneel (2010). Understanding Cryptography: A Textbook for Students and Practitioners. Springer. p. 7. ISBN 3642041000 